Dr Hima Hoskote, Dr Nazia ljaz, Dr Indira Srikantarajah (Lead for Acute Pain)

Luton and Dunstable University Hospital NHS Foundation Trust

\title{
Aims and Background
}

- To evaluate if post-operative Morphine PCA is essential at all.

- To propose acute pain protocol for Enhanced Recovery after Surgery (ERAS) for laparoscopic hysterectomy.

- ERAS was established to reduce morbidity and discomfort after operative procedures, proposing laparoscopic approaches to surgery to minimise postoperative acute pain, with the intension of minimising the use of Opioids.

This would then reduce nausea and vomiting in the postoperative period and reduce the hospital stay for patients, thus improving patient satisfaction.

Elements of Enhanced Recovery Pathway

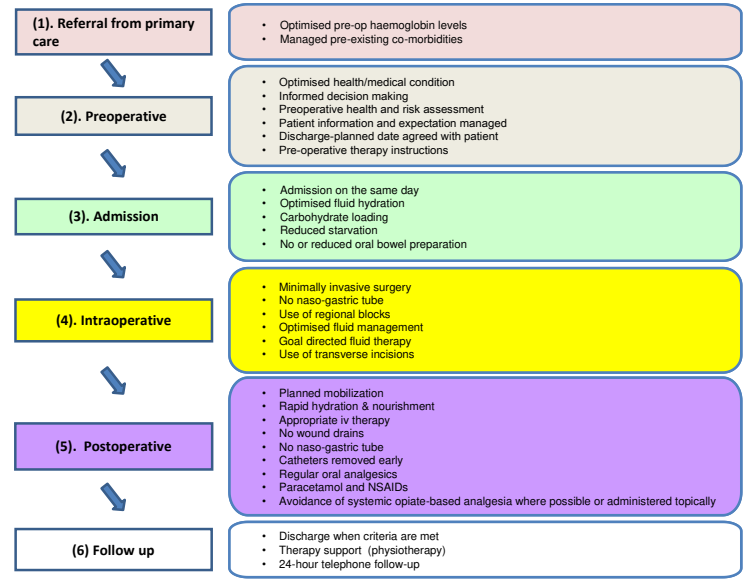

\section{Method}

- We looked at anaesthetic charts, post-operative pain charting on cases of laparoscopic hysterectomies over 6 months

- We aimed at a target of at least 20 cases, collected 21 cases
Parameters aimed at:

- Intra-operative cocktail of analgesics and anaesthetics

- Post-operative analgesics prescribed

- Pain assessment at different time intervals after surgery

\section{Results}

\begin{tabular}{|c|c|c|c|}
\hline Intra-operative & ANALGESICS GIVEN DURING SURGERY & \multicolumn{2}{|c|}{ NUMBER AND PERCENTAGES } \\
\hline \multirow{5}{*}{$\begin{array}{l}\text { Minimally invasive } \\
\text { surgery } \\
\text { Pain minimising } \\
\text { surgical approach } \\
\text { Avoidance of fluid } \\
\text { overload } \\
\text { Use of regional } \\
\text { anaesthetic } \\
\text { Short acting } \\
\text { anaesthetic agents } \\
\text { Hypothermia } \\
\text { prevention } \\
\text { VTE prophylaxis }\end{array}$} & 1) PARACETAMOL. & $(19 / 21)$ & $91 \%$ \\
\hline & 2) FENTANYL & $(17 / 21)$ & $81 \%$ \\
\hline & 3) NSAIDS & $(7 / 21)$ & $33 \%$ \\
\hline & 4) MORPHINE (INTERMITTENT) & $(17 / 21)$ & $81 \%$ \\
\hline & 5) OTHERS (e.g Remi Infusion) & $(6 / 21)$ & $29 \%$ \\
\hline
\end{tabular}

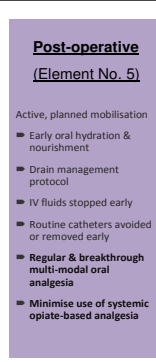

Pain Score at Different Time Intervals

\begin{tabular}{|c|c|c|c|}
\hline \multicolumn{2}{|c|}{ AT $6 \mathrm{Hrs}$} & \multicolumn{2}{|c|}{ AT $12 \mathrm{Hrs}$} \\
\hline $\begin{array}{l}\text { WITH } \\
\text { PCA }\end{array}$ & $\begin{array}{l}\text { NO } \\
\text { PCA }\end{array}$ & $\begin{array}{l}\text { WITH } \\
\text { PCA }\end{array}$ & $\begin{array}{l}\text { NO } \\
\text { PCA }\end{array}$ \\
\hline $20 \%$ & - & $5 \%$ & $5 \%$ \\
\hline $5 \%$ & $5 \%$ & $20 \%$ & $5 \%$ \\
\hline $30 \%$ & $15 \%$ & $20 \%$ & $10 \%$ \\
\hline $10 \%$ & $15 \%$ & $20 \%$ & $15 \%$ \\
\hline - & - & - & - \\
\hline
\end{tabular}

\begin{tabular}{|c|c|}
\hline \multicolumn{2}{|c|}{ AT 24 Hrs } \\
\hline WITH & NO \\
\hline PCA & PCA \\
\hline $15 \%$ & $5 \%$ \\
\hline $20 \%$ & $5 \%$ \\
\hline $20 \%$ & $10 \%$ \\
\hline $10 \%$ & $15 \%$ \\
\hline- & - \\
\hline
\end{tabular}

\begin{tabular}{|r|r|}
\hline \multicolumn{2}{|c|}{ ON DAY 3} \\
\hline \begin{tabular}{|r|r|} 
WITH \\
PCA
\end{tabular} & NO PCA \\
\hline $5 \%$ & $5 \%$ \\
\hline $5 \%$ & - \\
\hline $25 \%$ & $5 \%$ \\
\hline $5 \%$ & - \\
\hline $25 \%$ & $25 \%$ \\
\hline
\end{tabular}

\begin{tabular}{|c|c|c|}
\hline ANALGESICS PRESCRIBED POST-OPERATIVELY & NUMBER AND & PERCENTAGES \\
\hline 1) MORPHINEPCA & $(13 / 21)$ & $62 \%$ \\
\hline 2) MORPHINE INTERMITTENT & $(08 / 21)$ & $38 \%$ \\
\hline 3) OXYCODONEPCA & $(01 / 21)$ & $5 \%$ \\
\hline 4) PARACETAMOL & $(19 / 21)$ & $91 \%$ \\
\hline 5) NSAIDS & $(10 / 21)$ & $48 \%$ \\
\hline
\end{tabular}

Length of Stay

- Average LoS: 2.7 days - Max LoS: 7 days

Possible causes of delayed discharge

1. Inadequate pain relief

2. Post-op ileus

3. Post-Operative Nausea and Vomiting (PONV)

4. Infection/sepsis

5. Others: surgical causes

\section{Recommendations}

Suggestion for ER Pathway

Standardised Anaesthetic Protocol

- Standardised Anaesthetic Protocol (SAP) for Enhanced Recovery in laparoscopic hysterectomies

- Contribute to Implementation of the Enhanced Recovery (ER) protocol (anaesthetic component).

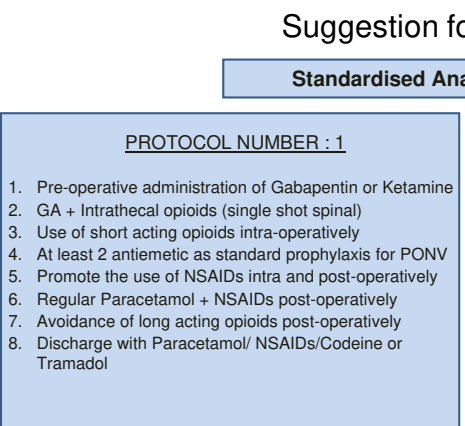




\section{PCA FOR LAPAROSCOPIC HYSTIERECTOMY - IS IT ESSENTIAL?}

\section{Dr. Nazia ljaz,Dr.Hima Hoskote, Dr. Indira \$rikantarajah (LEAD FOR ACUTE PAIN)}

\section{Aims}

- To evaluate if postoperative Morphine PCA is essential at all

- To propose acute pain protocol for Enhanced Recovery after Surgery (ERAS) for laparoscopic hysterectomy

\section{Background}

- ERAS got established to reduce morbidity and discomfort after operative procedures, proposing laparoscopic approaches to surgery to minimise

postoperative acute pain, with the intension of minimising the use of Opioids.

This would then reduce nausea and vomiting in the postoperative period and reduce the hospital stay for patients, thus improving patient satisfaction.

\section{Elements of enhanced recovery pathway}

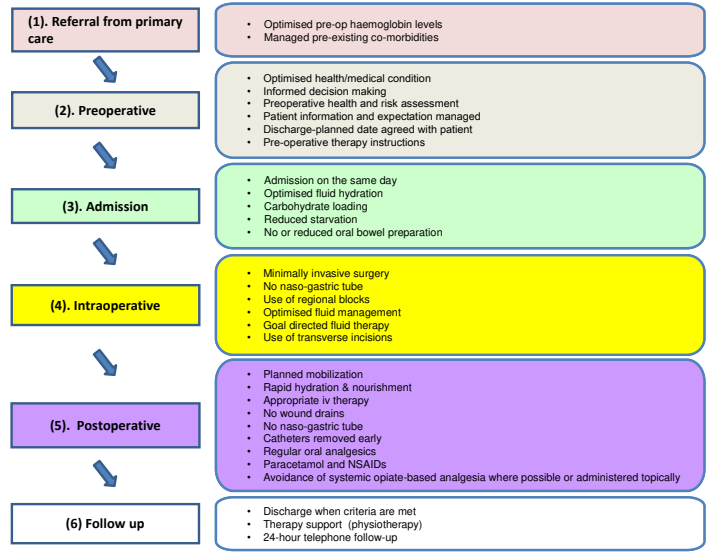

\section{Audit - Method}

Follow as many cases of laparoscopic hysterectomies over 6 months

- We aimed at a target of at least 20 cases

- Intra-operative cocktail of analgesics \& anaesthetics

- Post operative analgesics prescribed

- Pain assessment at different time intervals after surgery

- Looked at anaesthetic charts, post op pain charting

- Able to collect 21 cases

\section{Results}

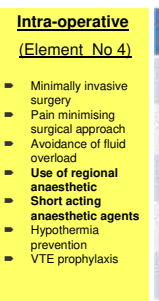

\begin{tabular}{|c|c|c|}
\hline ANALGESICS GIVEN DURING SURGERY & NUMBER AND PE: & RCENTAGES \\
\hline 1) PARACETAMOL & $(19 / 21)$ & $91 \%$ \\
\hline 2) FENTANYL & $(17 / 21)$ & $81 \%$ \\
\hline 3) NSAIDS & $(7 / 21)$ & $33 \%$ \\
\hline 4) MORPHINE (INTERMITTENT) & $(17 / 21)$ & $81 \%$ \\
\hline 5) OTHERS (e.g Remi Infusion) & $(6 / 21)$ & $29 \%$ \\
\hline
\end{tabular}

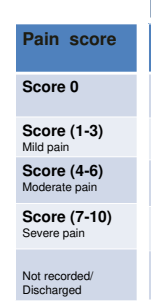

\begin{tabular}{|c|c|}
\hline \multicolumn{2}{|c|}{ AT 6 Hrs } \\
\hline $\begin{array}{c}\text { WITH } \\
\text { PCA }\end{array}$ & $\begin{array}{c}\text { NO } \\
\text { PCA }\end{array}$ \\
\hline $20 \%$ & - \\
\hline $5 \%$ & $5 \%$ \\
\hline $30 \%$ & $15 \%$ \\
\hline $10 \%$ & $15 \%$ \\
\hline- & - \\
\hline
\end{tabular}

\begin{tabular}{|c|c|}
\hline \multicolumn{2}{|c|}{ AT 12 Hrs } \\
\hline \begin{tabular}{c|c|} 
WITH \\
PCA
\end{tabular} & NO \\
\hline $5 \%$ & $5 \%$ \\
\hline $20 \%$ & $5 \%$ \\
\hline $20 \%$ & $10 \%$ \\
\hline $20 \%$ & $15 \%$ \\
\hline & - \\
\hline
\end{tabular}

\begin{tabular}{|c|c|}
\hline \multicolumn{2}{|c|}{ AT 24 Hrs } \\
\hline WITH & NO \\
\hline PCA & PCA \\
\hline $15 \%$ & $5 \%$ \\
\hline $20 \%$ & $5 \%$ \\
\hline $20 \%$ & $10 \%$ \\
\hline $10 \%$ & $15 \%$ \\
\hline- & - \\
\hline
\end{tabular}

\begin{tabular}{|r|r|}
\hline \multicolumn{2}{|c|}{ ON DAY 3} \\
\hline \begin{tabular}{|} 
WITH \\
PCA
\end{tabular} & NO PCA \\
\hline $5 \%$ & $5 \%$ \\
\hline $5 \%$ & - \\
\hline $25 \%$ & $5 \%$ \\
\hline $5 \%$ & - \\
\hline $25 \%$ & $25 \%$ \\
\hline
\end{tabular}

- $\quad$ Average LOS: 2.7 days

- Max LOS : 7 days

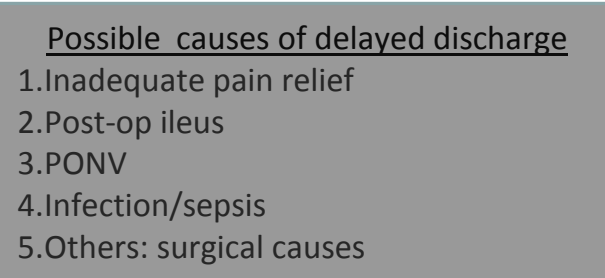

- Standardised Anaesthetic Protocol (SAP) for Enhanced Recovery in laparoscopic hysterectomies

- Contribute to Implementation of the Enhanced recovery protocol (anaesthetic component).

\begin{tabular}{|c|c|c|}
\hline & Standardised An & esthetic Protocol \\
\hline & PROTOCOL NUMBER : 1 & PROTOCOL NUMBER : 2 \\
\hline & Pre-operative administration of Gabapentin/Ketamine & 1. Pre-operative administration of Gabapentin/Ketamine \\
\hline & GA + Intrathecal opioids (single shot spinal) & 2. GA + Truncal nerve blocks ( TAP/ illio-inguinal b/L) \\
\hline & Use of short acting opioids intra-operatively & 3. Use of short acting opioids intra-operatively \\
\hline & $\begin{array}{l}\text { At least } 2 \text { antiemetic as standard prophylaxis for } \\
\text { PONV }\end{array}$ & $\begin{array}{l}\text { 4. At least } 2 \text { antiemetic as standard prophylaxis for } \\
\text { PONV }\end{array}$ \\
\hline & Promote the use of NSAIDs intra and post operatively & 5. Promote the use of NSAIDs intra and post operatively \\
\hline & Regular Paracetamol + NSAIDs post op & 6. Regular Paracetamol + NSAIDs post op \\
\hline & Avoidance of long acting opioids post operatively & 7. Avoidance of long acting opioids post operatively \\
\hline & Discharge with Paracetamol/ NSAIDs/ Codeine or & $\begin{array}{l}\text { 8. Discharge with Paracetamol/ NSAIDs/ Codeine or } \\
\text { Tramadol }\end{array}$ \\
\hline
\end{tabular}

Vecchio, R., MacFayden, B.V. and Palazzo, F., 2000. History of laparoscopic surgery. Panminerva medica, 42(1), pp.87-90.

Gustafsson, U.O., Scott, M.J., Schwenk, W., Demartines, N., Roulin, D., Francis, N., McNaught,

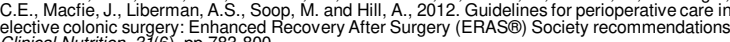
Clinical Nutrition, $37(6)$, pp.783-800.

ERAS Guidelines- Association of Surgeons of Great Britain and Ireland. Guidelines for
Implementation of Enhanced Recovery Protocols. 2009. www. asgbi.org.uk (accessed 02/05/2016) www.erassociety.org

www.erassociety.org
Simpson, J.C.
Galswoon Mosinghe, S.R., Grocott, M.P.W., Kuper, M. McMeeking, A., Oliver, C.M., the enhanced recovery partnership programme 2009-2012. British journal of anaesthesia, p.aev105. 